OPEN ACCESS

Edited by:

Daniel Giddings Vassão, Max-Planck-Institut für chemische

Ökologie, Germany

Reviewed by:

Luis Sampedro,

Consejo Superior de Investigaciones

Cientificas (CSIC), Spain

Maria Pappas,

Democritus University of Thrace,

Greece

*Correspondence:

Heather L. Slinn

heather.slinn@gmail.com

Specialty section:

This article was submitted to

Plant Metabolism

and Chemodiversity,

a section of the journal

Frontiers in Plant Science

Received: 05 February 2018

Accepted: 27 April 2018

Published: 11 June 2018

Citation:

Slinn HL, Richards LA, Dyer LA, Hurtado PJ and Smilanich AM (2018) Across Multiple Species,

Phytochemical Diversity

and Herbivore Diet Breadth Have

Cascading Effects on Herbivore Immunity and Parasitism in a Tropical

Model System.

Front. Plant Sci. 9:656.

doi: 10.3389/fpls.2018.00656

\section{Across Multiple Species, Phytochemical Diversity and Herbivore Diet Breadth Have Cascading Effects on Herbivore Immunity and Parasitism in a Tropical Model System}

\author{
Heather L. Slinn*, Lora A. Richards, Lee A. Dyer, Paul J. Hurtado and \\ Angela M. Smilanich
}

\section{Department of Biology, University of Nevada, Reno, Reno, NV, United States}

Terrestrial tri-trophic interactions account for a large part of biodiversity, with approximately $75 \%$ represented in plant-insect-parasitoid interactions. Herbivore diet breadth is an important factor mediating these tri-trophic interactions, as specialisation can influence how herbivore fitness is affected by plant traits. We investigated how phytochemistry, herbivore immunity, and herbivore diet breadth mediate plantcaterpillar-parasitoid interactions on the tropical plant genus Piper (Piperaceae) at $\mathrm{La}$ Selva Biological station in Costa Rica and at Yanayacu Biological Station in Ecuador. We collected larval stages of one Piper generalist species, Quadrus cerealis, (Lepidoptera: Hesperiidae) and 4 specialist species in the genus Eois (Lepidoptera: Geometridae) from 15 different species of Piper, reared them on host leaf material, and assayed phenoloxidase activity as a measure of potential larval immunity. We combined these data with parasitism and caterpillar species diet breadth calculated from a 19-year database, as well as established values of phytochemical diversity calculated for each plant species, in order to test specific hypotheses about how these variables are related. We found that phytochemical diversity was an important predictor for herbivore immunity, herbivore parasitism, and diet breadth for specialist caterpillars, but that the direction and magnitude of these relationships differed between sites. In Costa Rica, specialist herbivore immune function was negatively associated with the phytochemical diversity of the Piper host plants, and rates of parasitism decreased with higher immune function. The same was true for Ecuador with the exception that there was a positive association between immune function and phytochemical diversity. Furthermore, phytochemical diversity did not affect herbivore immunity and parasitism for the more generalised herbivore. Results also indicated that small differences in herbivore diet breadth are an important factor mediating herbivore immunity and parasitism success for Eois at both sites. These patterns contribute to a growing body of literature that demonstrate strong cascading effects of phytochemistry on higher trophic 
levels that are dependent on herbivore specialisation and that can vary in space and time. Investigating the interface between herbivore immunity, plant chemical defence, and parasitoids is an important facet of tri-trophic interactions that can help to explain the enormous amount of biodiversity found in the tropics.

Keywords: tropics, Piper, tri-trophic interactions, phytochemical diversity, parasitism, diet breadth, chemodiversity

\section{INTRODUCTION}

Tri-trophic interactions are an important feature of biotic communities and contribute to the maintenance of biodiversity as well as mediate ecosystem processes (Price et al., 1980; Hunter and Price, 1992; Agrawal, 2000; Price, 2002; Whitham et al., 2006). For instance, terrestrial plant-insect-predator/parasitoid interactions may make up approximately three quarters of the diversity of multicellular organisms (Price, 2002). Ecologists have found that tri-trophic interactions can shape community parameters, such as species diversity, functional diversity, primary productivity, and consumer abundance (Hairston et al., 1960; Ives et al., 2005; Singer and Stireman, 2005; Crutsinger et al., 2006; Johnson, 2008; O'Connor et al., 2016). Many tri-trophic studies have focused on how primary producers affect biotic communities through effects on densities or population dynamics of herbivores, mutualists, and natural enemies (Crutsinger et al., 2006; Crawford et al., 2007; Barbour et al., 2015). Plant chemical defence is one of the most important components of these bottom-up effects, and there is a rich literature documenting how chemistry affects plant-insect interactions (Fraenkel, 1959; Ehrlich and Raven, 1964; Schoonhoven et al., 2005; Hunter, 2016), via both negative and positive physiological and behavioural effects on herbivores and natural enemies (Smilanich et al., 2016). One clear gap in our knowledge of how phytochemistry influences tri-trophic interactions is empirical data that consider the entire suite of plant secondary metabolites in a species instead of focusing on one or two major compounds (Richards et al., 2010, 2016; Smilanich et al., 2016). Given that herbivores are exposed to the full array of compounds during their larval development and as adults, significant consideration should be given to the diversity of secondary metabolites found in plants (Hay et al., 1994; Richards et al., 2015). Here, we use phytochemical diversity as a metric of plant defence to investigate the effects on herbivore performance as measured by immune strength, and whether effects on the immune response cascade to impact parasitism success (Smilanich et al., 2009b; Richards et al., 2015; Hansen et al., 2017).

Research on the role of herbivore immunity as a mediator of tri-trophic interactions has been expanding over the last decade (Bukovinszky et al., 2009; Smilanich et al., 2009a; Richards et al., 2012; Singer et al., 2014; Lampert and Bowers, 2015). However, the majority of this work has been performed in temperate systems (but see: Smilanich et al., 2009b; Smilanich and Dyer, 2012; Hansen et al., 2017), where plant chemistry is typically less diverse and compounds may be less toxic (Coley and Barone, 1996; Dyer and Coley, 2002). In general, increased concentrations or mixture complexities of plant chemical compounds have a detrimental impact on herbivore immunity (Haviola et al., 2007; Smilanich et al., 2009a; Richards et al., 2010, 2016; Lampert, 2012; Hansen et al., 2017), but these effects can differentially influence the success of predators and parasitoids (Dyer et al., 2004; Bukovinszky et al., 2009; Richards et al., 2015). For instance, specialist caterpillars (Junonia coenia: Nymphalidae) have a weakened immune response due to sequestering higher concentrations of secondary metabolites, and this has been termed the 'vulnerable host hypothesis' (Smilanich et al., 2009a; Lampert and Bowers, 2015). More generally, specialised herbivores should be better adapted to diverse mixtures of secondary metabolites in their specific host plants, which may also protect specialists from natural enemies (e.g., Dyer, 1995); however, the energetic costs that accompany sequestration may be toxic to immune cells or may lead to reallocation of resources away from immune functions, rendering specialists more susceptible to parasitism (Smilanich et al., 2009a). Chemically defended or immune-compromised specialists may provide a 'safe haven' for parasitoids because they are less likely to be attacked or consumed by other natural enemies, which tend to avoid toxic specialist hosts (Dyer, 1995). Indeed, generalists are often better protected than specialists against parasitoids (Dyer and Gentry, 1999). The vulnerable host and safe haven hypotheses suggest that phytochemically defended plants may host specialist herbivores that are immunocompromised and more likely to be attacked by parasitoids (Smilanich et al., 2009a; Lampert et al., 2010).

The effect of host plant chemistry on the immune response also depends on the physiological ecology of the organism: herbivores that utilise metabolically expensive strategies, such as detoxification or sequestration, to tolerate host plant chemistry may incur physiological costs to eating toxic diets and experience compromised immune systems (Smilanich et al., 2009a). For example, the immune response of Eois nympha and Eois apyraria (Geometridae) caterpillars was suppressed when feeding on Piper cencocladum (Piperaceae) compared to other Piper host plants, and $P$. cenocladum is more phytochemically diverse than other Piper host species (i.e., Piper imperiale) (Hansen et al., 2017). Furthermore, Richards et al. (2010) found that a mixture of plant secondary metabolites from a neotropical shrub in the genus Piper (Piperaceae) affected a naïve generalist noctuid caterpillar (Spodoptera) differently from adapted specialist geometrid caterpillars (Eois), with Spodoptera experiencing high mortality through direct toxicity, and indirect negative effects of chemistry on Eois via increased levels of parasitism. Increased parasitism associated with host plant toxicity is also consistent with the hypothesis that higher phytochemical diversity may weaken a caterpillar's immune response, leading to increased parasitoid 
success. This hypothesised association is best tested when direct effects of chemistry on adult parasitoids are ruled out, which is the case in experiments where caterpillars are naturally exposed to parasitoids first in the field and then subsequently assigned to feeding treatments in the laboratory (e.g., Smilanich et al., 2009b; Richards et al., 2010; Hansen et al., 2017). Similarly, iridoid glycosides sequestered by buckeye caterpillars (J. coenia) negatively affected the efficacy of encapsulation by these specialists (Smilanich et al., 2009a) but did not affect this same (encapsulation) measure of the immune response in the generalist caterpillar, Grammia incorrupta (Erebidae: Arctiinae) (Smilanich et al., 2011). Overall, there is growing evidence that plant chemistry may mediate herbivore susceptibility to parasitoids via the herbivore's immunity and the strength or direction of this relationship is dependent on the level of specialisation of the plant-herbivore interaction. While previous studies have included how diet breadth may affect the ecoimmunology of tri-trophic interactions, there are other axes of variation that are likely to be important for modifying this relationship, including biogeographical differences among sites. For example, plant chemistry and tri-trophic interactions vary across elevations (Rodríguez-Castañeda et al., 2016) and with rainfall intensity (Cunningham et al., 1999), thus the same herbivore species may be affected differently by host plant chemistry and parasitoids across elevational and precipitation gradients, due to differences in chemistry and in enemy communities (Rodríguez-Castañeda et al., 2016).

In this study, we used the tropical plant genus, Piper, the associated specialist herbivore genus, Eois (Lepidoptera: Geometridae), and a Piper generalist, Quadrus cerealis (Lepidoptera: Hesperiidae), to investigate whether variation in phytochemical diversity influences the strength of the herbivore immune response and associated levels of parasitism (Figure 1 and Table 1). In addition to examining variation across these different herbivore species, we examined these relationships in two distinct ecosystems - a lowland wet forest in Costa Rica (La Selva, Sarapiqui) and a cloud forest in Ecuador (Yanayacu, Napo), which differ dramatically in temperature means and variance, annual rainfall, and elevation. Specifically, we designed our study to address the following questions: (1) How does phytochemical diversity influence herbivore immunity and levels of parasitism and how are these relationships affected by diet breadth? (2) How do these effects vary across different herbivore species and different locations?

\section{MATERIALS AND METHODS}

\section{Study Sites}

Our study took place at two different field stations in the neotropics: (1) La Selva Biological Station, Heredia Province, Costa Rica ( $\left.10^{\circ} 26^{\prime} \mathrm{N} 83^{\circ} 59^{\prime} \mathrm{W}\right)$ and (2) Yanayacu Biological station, Napo Province, Ecuador $\left(00^{\circ} 36^{\prime} \mathrm{S} 77^{\circ} 53^{\prime} \mathrm{W}\right)$. The La Selva Biological reserve is 1600 ha of lowland rainforest and ranges from 35 to $140 \mathrm{~m}$ in elevation and is surrounded by a combination of disturbed, agricultural habitat, and natural forest. The mean annual precipitation is approximately $4200 \mathrm{~mm}$.

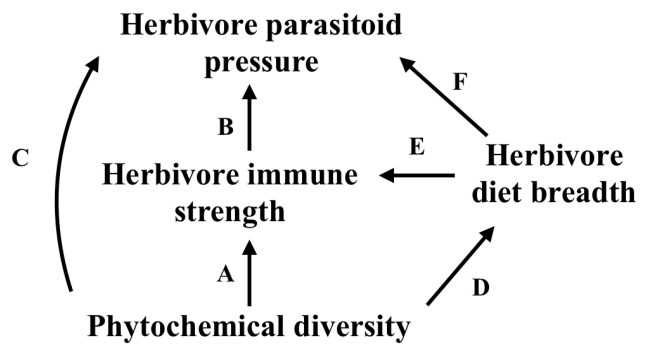

FIGURE 1 | Meta-model that structured our a priori hypotheses. Letters over paths are associated with hypotheses in Table $\mathbf{1 .}$

Sampling at Yanayacu Biological Station included the 100 ha owned by the station as well as thousands of hectares of surrounding cloud forest on the slopes of the eastern Andes. The elevation at the station is $2100 \mathrm{~m}$ and the annual precipitation is approximately $2624 \mathrm{~mm}$.

\section{Piper-Eois, Piper-Quadrus System}

The plant genus Piper (Piperaceae) is an emerging tropical model system for studying tri-trophic interactions because of the growing knowledge on its evolutionary history, genomics, plant chemistry, distribution, and insect communities (Marquis, 1991; Greig, 1993; Dyer and Palmer, 2004; Richards et al., 2015; Glassmire et al., 2016; Salazar et al., 2016). Currently there are over 2000 species of Piper that have been identified pantropically, with approximately 1300 species occurring in the neotropics, 50 species present at the La Selva Biological station and 20 present at the Yanayacu station. Piper is a phytochemically diverse genus, including compounds from at least 15 classes, and a total of 667 individual compounds have been discovered (Richards et al., 2016). In this study, we used previously published data quantifying phytochemical diversity for multiple Piper species (Richards et al., 2015). Each of the Piper species in this experiment had a fixed diversity value and therefore no intra-specific variation was quantified. Briefly, phytochemical diversity is an effective number of functional groups, transformed from a Simpson's diversity entropy calculated from proton nuclear magnetic resonance $\left({ }^{1} \mathrm{H}-\mathrm{NMR}\right)$, which incorporates both mixture complexity and structurally complexity, the two key components of chemical diversity (Richards et al., 2015).

Piper species host diverse lepidopteran herbivore communities that vary in diet breadth (Dyer and Palmer, 2004). Caterpillars in the genus Eois (Lepidoptera: Geometridae) are Piper specialists that feed exclusively on 1-4 different Piper species (Connahs et al., 2009). They are one of the most well studied and abundant genera of caterpillars found on Piper, and over $80 \%$ of Eois species are found in the neotropics with others in Africa, Asia, and Australia (Rodríguez-Castañeda et al., 2010; Brehm et al., 2011). In contrast, the Piper skipper, Q. cerealis (Lepidoptera: Hesperiidae), has been recorded feeding on 23 Piper species; in this paper we categorise this skipper as a Piper generalist $^{1}$ (Dyer et al., 2010).

\footnotetext{
${ }^{1}$ http://www.caterpillars.org
} 
TABLE 1 | Description of the hypotheses and predictions behind each path in our supported SEM models.

\begin{tabular}{|c|c|c|c|c|}
\hline Explanatory variables & $\begin{array}{l}\text { Response } \\
\text { variables }\end{array}$ & Paths & Hypotheses and predictions & References \\
\hline $\begin{array}{l}\text { Plant quality (phytochemical } \\
\text { diversity) }\end{array}$ & $\begin{array}{l}\text { Herbivore fitness } \\
\text { (Immunity) }\end{array}$ & A & $\begin{array}{l}\text { Plants with high phytochemical diversity are more likely } \\
\text { to contain compounds that decrease herbivore fitness. }\end{array}$ & $\begin{array}{l}\text { Berenbaum and Neal, 1985; Jones } \\
\text { and Firn, 1991; Smilanich et al., } \\
\text { 2009b; Diamond and Kingsolver, } \\
\text { 2011; Lampert and Bowers, } 2015\end{array}$ \\
\hline Herbivore fitness (immunity) & $\begin{array}{l}\text { Herbivore } \\
\text { parasitism }\end{array}$ & $B$ & $\begin{array}{l}\text { The immune system provides important protection } \\
\text { against parasitoids, thus as the strength of the immune } \\
\text { system decreases, parasitism increases. }\end{array}$ & $\begin{array}{l}\text { Bukovinszky et al., 2009; Smilanich } \\
\text { et al., 2009a; Quintero et al., } 2014\end{array}$ \\
\hline $\begin{array}{l}\text { Plant quality (phytochemical } \\
\text { diversity) }\end{array}$ & $\begin{array}{l}\text { Herbivore } \\
\text { parasitism }\end{array}$ & $\mathrm{C}$ & $\begin{array}{l}\text { Low plant quality caused by toxic secondary } \\
\text { metabolites, and higher phytochemical diversity are } \\
\text { more likely to weaken herbivores via the presence of } \\
\text { bioactive compounds and/or toxic synergies, increasing } \\
\text { parasitoid success. }\end{array}$ & $\begin{array}{l}\text { Lill et al., 2002; Bukovinszky et al., } \\
\text { 2009; Richards et al., 2010; } \\
\text { Sternberg et al., 2012; Hunter, } \\
2016\end{array}$ \\
\hline $\begin{array}{l}\text { Plant quality (phytochemical } \\
\text { diversity) }\end{array}$ & $\begin{array}{l}\text { Herbivore diet } \\
\text { breadth }\end{array}$ & $\mathrm{D}$ & $\begin{array}{l}\text { Plants with greater diversity of phytochemical } \\
\text { compounds are more likely to host specialised } \\
\text { herbivores that have adapted to bioactive compounds } \\
\text { and/or toxic synergies. }\end{array}$ & $\begin{array}{l}\text { Becerra, 2007, 2015; Dyer et al., } \\
\text { 2003, 2007; Richards et al., } 2015\end{array}$ \\
\hline Herbivore diet breadth & $\begin{array}{l}\text { Herbivore fitness } \\
\text { (immunity) }\end{array}$ & $E$ & $\begin{array}{l}\text { Specialist herbivores are adapted to detoxifying or } \\
\text { sequestering toxic plant compounds and will perform } \\
\text { better on their host plants than generalists. }\end{array}$ & $\begin{array}{l}\text { Coley et al., 2006; Richards et al., } \\
\text { 2010; Lampert, } 2012\end{array}$ \\
\hline Herbivore diet breadth & $\begin{array}{l}\text { Herbivore } \\
\text { parasitism }\end{array}$ & $\mathrm{F}$ & $\begin{array}{l}\text { Herbivores that feed on a greater number of plants are } \\
\text { exposed to a greater variety of toxic plant compounds } \\
\text { which weaken herbivores, increasing parasitoid } \\
\text { success. }\end{array}$ & $\begin{array}{l}\text { Barbosa et al., 1991; Carvalheiro } \\
\text { et al., 2010; Lampert et al., 2011; } \\
\text { Reudler et al., } 2011\end{array}$ \\
\hline
\end{tabular}

\section{Long Term Rearing Databases}

Since 1991, principal investigators, students, volunteers, and technicians have been collecting plant-herbivore-parasitism interaction data in Costa Rica (e.g., from Dyer and Floyd, 1993; Hansen et al., 2017). We used data from 1996 to 2015 in this database (these years included the most complete parasitism data) to determine simple taxonomic diet breadth for herbivores (number of host plants documented for a caterpillar species) and parasitism frequency, quantified as the total number of parasitized caterpillars divided by the total number of caterpillars reared to adult plus parasitoid (parasitoids)/(healthy adults + parasitoids) (Gentry and Dyer, 2002; Hansen et al., 2017; Tables 2, 3). Data consisted mainly of entries from La Selva Biological Station, but also from other areas nearby such as Braulio Carrillo National Park and the Tirimbina Biological Reserve. Primarily third instar caterpillars were collected yearround in all forest types and reared on the host plant from which they were collected in ambient conditions until they pupated and eclosed into adulthood, or if parasitized prior to collection, until they succumbed to parasitism. Data were collected on the caterpillar species, the host plant it was found on, and whether it reached adulthood or was parasitized (for detailed methods see Gentry and Dyer, 2002). Using these data, we evaluated herbivore immunity for four different Eois species collected from five different Piper species (Table 2). For these species, we found a total of 2011 records in our database with 900 caterpillars successfully reaching adulthood (Table 2). Additionally, we collected Q. cerealis from 10 different Piper species, though we have records of larvae feeding on 23 different Piper species (Table 3). We recorded 117 instances of Q. cerealis on these 10 Piper species with 75 caterpillars successfully reaching adulthood
(Table 3). Tables 2, 3 summarise the sample sizes of larvae collected for immune assays and long-term parasitism sample sizes for those same species.

The same data collection procedure was utilised at the Ecuador site, where the database spans 15 years (2001-2015). Larvae were collected in the cloud forest surrounding Yanayacu Biological Station. At this site, we measured the immune response from eight Eois morphospecies feeding on three different Piper species (Table 2). We had 2079 records of our Eois morphospecies in our database with 809 caterpillars successfully reaching adulthood (Table 2). We calculated diet breadth and levels of parasitism using the same method at both sites. Diet breadth was calculated as the number of Piper species on which a caterpillar species was found feeding and successfully reared to adult moth or parasitoid. As with the Costa Rica data, parasitism frequency was calculated as the number of parasitism events for each caterpillar species divided by the total number of successfully reared adults + parasitoids.

\section{Immune Assay}

Phenoloxidase (hereafter PO) is an important enzyme for triggering the melanization process, a mechanism of innate immunity involving deposition of pigments on foreign bodies (Beckage, 2008; González-Santoyo and Córdoba-Aguilar, 2012). It is typically stored in hemolymph cells in a non-activated form called prophenoloxidase (proPO) since active PO can have locally toxic effects (Cerenius et al., 2008). Upon infection or natural enemy attack, proPO is converted to the active form, PO, which catalyses the cascade to produce melanin. Phenoloxidase has been shown to be an important part of the immune response in arthropods, protecting them from 
TABLE 2 | Eois caterpillars and their host plants collected for immune assays.

\begin{tabular}{|c|c|c|c|c|c|c|}
\hline \multirow[t]{2}{*}{ Site } & \multirow[t]{2}{*}{ Eois spp. } & \multirow[t]{2}{*}{ Piper spp. } & \multirow[b]{2}{*}{$n$} & \multicolumn{2}{|c|}{ Database } & \multirow[b]{2}{*}{$\%$ parasitized } \\
\hline & & & & Records & Adults & \\
\hline \multirow[t]{6}{*}{ Costa Rica } & Eois nympha & Piper biseriatum & 9 & 44 & 7 & 29 \\
\hline & & Piper cenocladum & 28 & 921 & 317 & 18 \\
\hline & Eois apyraria & Piper cenocladum & 1 & 328 & 164 & 8.4 \\
\hline & & Piper imperiale & 7 & 616 & 359 & 1.4 \\
\hline & Eois russearia & Piper sancti-felicis & 12 & 48 & 24 & 4 \\
\hline & Eois mexicaria & Piper umbricola & 13 & 54 & 29 & 0 \\
\hline Total & & & 70 & 2011 & 900 & \\
\hline \multirow[t]{14}{*}{ Ecuador } & Six black two pink spots & Piper baezanum & 2 & 6 & 1 & 0 \\
\hline & & Piper kelleyi & 16 & 1792 & 700 & 14 \\
\hline & & Piper lancifolium & 1 & 1 & 0 & 0 \\
\hline & Lime slime & Piper baezanum & 1 & 3 & 1 & 0 \\
\hline & & Piper kelleyi & 7 & 9 & 0 & 0 \\
\hline & Two black spots & Piper kelleyi & 27 & 83 & 29 & 3.3 \\
\hline & & Piper lancifolium & 1 & 1 & 0 & 0 \\
\hline & Eois viridiflava Dognin & Piper baezanum & 1 & 2 & 0 & 0 \\
\hline & & Piper lancifolium & 20 & 36 & 0 & 0 \\
\hline & Pink spots funk & Piper kelleyi & 3 & 86 & 37 & 8.1 \\
\hline & & Piper lancifolium & 1 & 1 & 0 & 0 \\
\hline & Eight black blur & Piper baezanum & 1 & 1 & 9 & 0 \\
\hline & Eois beebei Fletcher & Piper kelleyi & 1 & 36 & 19 & 14 \\
\hline & Eois ignefumataPdfLatex Dognin & Piper kelleyi & 1 & 22 & 13 & 19 \\
\hline Total & & & 83 & 2079 & 809 & \\
\hline
\end{tabular}

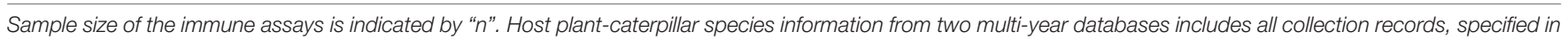
the 'records' column, caterpillars that made it to adulthood and parasitism percentage.

TABLE 3 | Quadrus cerealis caterpillars and their host plants collected for immune assays.

\begin{tabular}{|c|c|c|c|c|c|}
\hline \multirow[t]{2}{*}{ Site } & \multirow[t]{2}{*}{ Piper spp. } & \multirow[b]{2}{*}{$n$} & \multicolumn{2}{|c|}{ Database } & \multirow[b]{2}{*}{$\%$ parasitized } \\
\hline & & & Records & Adults & \\
\hline \multirow[t]{10}{*}{ Costa Rica } & Piper arboreum & 3 & 2 & 2 & 0 \\
\hline & Piper cenocladum & 1 & 4 & 3 & 25 \\
\hline & Piper colonense & 13 & 16 & 13 & 38 \\
\hline & Piper garagaranum & 1 & 3 & 2 & 33 \\
\hline & Piper imperiale & 6 & 2 & 1 & 50 \\
\hline & Piper multiplinervium & 19 & 26 & 26 & 7.7 \\
\hline & Piper pseudobumbratum & 1 & 1 & 1 & 0 \\
\hline & Piper reticulatum & 18 & 62 & 26 & 68 \\
\hline & Piper trigonum & 2 & 0 & 0 & 0 \\
\hline & Piper umbricola & 1 & 1 & 1 & 0 \\
\hline Total & & 65 & 117 & 75 & \\
\hline
\end{tabular}

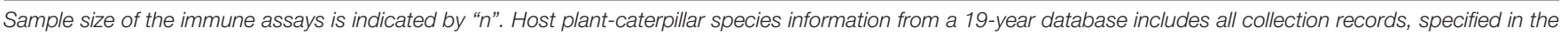
'records' column, caterpillars that made it to adulthood and parasitism percentage.

bacteria, viruses, and parasitoids (Cerenius et al., 2008). We measured the activity of the PO enzyme as an indicator of the strength of the herbivore immune response (González-Santoyo and Córdoba-Aguilar, 2012). We collected four species of early instar caterpillars from five different plant species and reared them on the host plant in which they were found in ambient conditions until they reached 5th instar. To measure PO activity (modified from Adamo, 2004), we took $2 \mu \mathrm{L}$ of hemolymph from each Eois caterpillar (Costa Rica: $N=70$, Ecuador: $N=83$ ) and $5 \mu \mathrm{L}$ from each Q. cerealis caterpillar (Costa Rica: $N=65$ ), collected by puncturing the caterpillar with a pin and extracting hemolymph with a pipette. The volume of hemolymph was 
divided the into two Eppendorf tubes-one for cell-free PO found in the hemolymph at the time the hemolymph is taken (standing PO), and one for cell-bound PO, which is artificially activated by adding a chemical activator (total PO). The aliquots of hemolymph were added to $50 \mu \mathrm{l}$ of phosphate buffered saline for Eois individuals and $100 \mu \mathrm{l}$ PBS for Q. cerealis individuals. For the total PO in both species, $35 \mu \mathrm{l}$ of chymotrypsin $(1 \mathrm{mg} / \mathrm{mL})$ was added to the PBS-bound hemolymph, vortexed for $2 \mathrm{~s}$, then incubated at room temperature for $20 \mathrm{~min}$. During incubation, the substrate, dopamine, $(0.0284 \mathrm{~g} / 10 \mathrm{~mL}$ distilled water $)$ was prepared. Since this compound is light sensitive, fresh dopamine was prepared daily. For Eois, we added $300 \mu$ l of dopamine to each Eppendorf tube, vortexed for $2 \mathrm{~s}$, then added $25 \mu \mathrm{l}$ of the dopamine-hemolymph mixture to a well plate. For Q. cerealis, we added $500 \mu \mathrm{l}$ of dopamine to each Eppendorf tube, vortexed for $2 \mathrm{~s}$, then added $200 \mu \mathrm{l}$ of the dopamine-hemolymph mixture to a well plate. We used a spectrophotometer (BIO-RAD: iMark Microplate Absorbance Reader) at a wavelength of $490 \mathrm{~nm}$ to measure the activity of PO every $30 \mathrm{~s}$ for $45 \mathrm{~min}$. We measured the slope, which was the rate of reaction, from the first $10 \mathrm{~min}$ because it was a linear increase. PO assays were performed in Costa Rica from January 2013 to December 2015 and in Ecuador from December 2015 to January 2016.

\section{Statistical Analyses}

We used structural equation models (SEM) to evaluate 7 a priori hypotheses, which tested for bottom-up effects of phytochemical diversity and herbivore diet breadth on herbivore immunity and parasitism success (Figure $\mathbf{1}$ and Table 1). We used the global estimation method in the R packages piecewiseSEM v.1.2.1 (Lefcheck, 2016) and lavaan v.0.5-23 to run our SEMs (Rosseel et al., 2017) in R v3.4.2 (R Core Team, 2017). We were not able to normalise the residuals of our data, so we chose a more robust estimator to account for non-normality and unequal variance instead of the default maximum likelihood method; this method is based on the Satterthwaite approach and is called the maximum likelihood estimation with robust standard errors and a mean and variance adjusted test statistic (Rosseel et al., 2017). Lastly, we used the same 7 hypotheses in our Ecuador dataset as we had no reason to believe that our systems should operate differently (Figure 1 and Table 1).

For each site, we used a Bayesian mixed linear model to examine effects of phytochemical diversity on immune response. This approach allowed us to incorporate prior distributions from earlier studies using the same methodology (Smilanich and Dyer, 2012), also to account for Type II error (i.e., reporting actual probabilities of null hypotheses) and to test the generalizability of our results. Caterpillar species were a random effect in the model. Priors were generated from E. nympha and E. apyraria caterpillars collected on $P$. biseriatum, P. cenocladum, $P$. imperiale, and P. urostachyum at La Selva Biological Station in Costa Rica. The Bayesian model was estimated using SAS 9.4 (v13.1) procedure MCMC. We chose the quasi-Newton algorithm, convergence was assessed via visual examination of the trace plot, and the first 2,000 (burn-in) out of 10,000 samples were discarded, yielding robust posterior distributions for parameters. We report the posterior distributions of $B_{1}$ parameter estimates from this model for the effects of phytochemical diversity on rate of total PO absorbance per minute.

\section{RESULTS}

\section{Summary Statistics}

Average immune response for Eois, as measured by total PO absorbance per minute ( $\triangle \mathrm{Abs}$ ), was approximately equal across sites (Eois: Costa Rica: $0.03 \pm 0.004 \Delta$ Abs; Ecuador: $0.02 \pm 0.001 \triangle \mathrm{Abs}$; here and elsewhere, error is 1 SEM), and between specialist Eois and Piper generalist, Q. cerealis (Q. cerealis: $0.02 \pm 0.002 \Delta \mathrm{Abs})$. However, average parasitism level was higher for $Q$. cerealis ( $0.34 \pm 0.03$ parasitism frequency) compared to Eois at both sites (Costa Rica: $0.12 \pm 0.01$ parasitism frequency; Ecuador: $0.04 \pm 0.01$ parasitism frequency). Parasitoid families attacking the caterpillars also differed between sites and species. Q. cerealis parasitism was entirely tachinid parasitoids, while Eois parasitism in Costa Rica was $80 \%$ braconids, $8 \%$ tachinids, and $12 \%$ parasitism by other families. Eois parasitism in Ecuador was $24 \%$ tachinids, $41 \%$ braconids, and 35\% parasitism by other families. Increases in phytochemical diversity had negative effects on the immune response in both Costa Rica and Ecuador, with posterior distributions of parameter estimates (from the mixed Bayesian model) similar to those reported previously for effects of diet on immune response. The negative effects of phytochemical diversity on immune response yielded parameter estimates at both sites that did not include a slope of zero; the combined mean slope for effect of NMR bin diversity on $\Delta$ Abs was -0.46 .

\section{Structural Equation Models}

Overall, the best fit structural equation models supported the hypotheses that both phytochemical diversity and herbivore diet breadth are important factors shaping herbivore immunity and parasitism for Eois species in both Ecuador and Costa Rica, however, for some relationships, the directions of the effects were reversed from one site to another (Table 4 and Figures 2, 3). Tests of seven a priori models to explain the relationships of our measured variables were completed for both sites. In addition, we tested our models by bootstrapping missing data to even out sampling effort (see Supplementary Tables S1, S2). This analysis yielded only one of the same models as our initial analysis without the bootstrapped data for Costa Rica but not Ecuador (Model II, the diet breadth regulation hypothesis; Supplementary Table S3).

\section{Model I: Phytochemical Diversity Regulation Hypothesis}

The phytochemical diversity regulation hypothesis (Model I) for our Costa Rica Eois data included phytochemical diversity as an exogenous variable with direct paths to herbivore immunity, herbivore diet breadth, and herbivore parasitism; the model also included effects of herbivore immunity and diet breadth on herbivore parasitism (Costa Rica model fit: Robust test statistic $=0.004, \mathrm{df}=1, P=0.95$, scaling factor $=2.08)$. This model supported the hypothesis that there is a strong direct 
TABLE 4 | Structural equation model (SEM) results from Costa Rica Eois and Q. cerealis study systems.

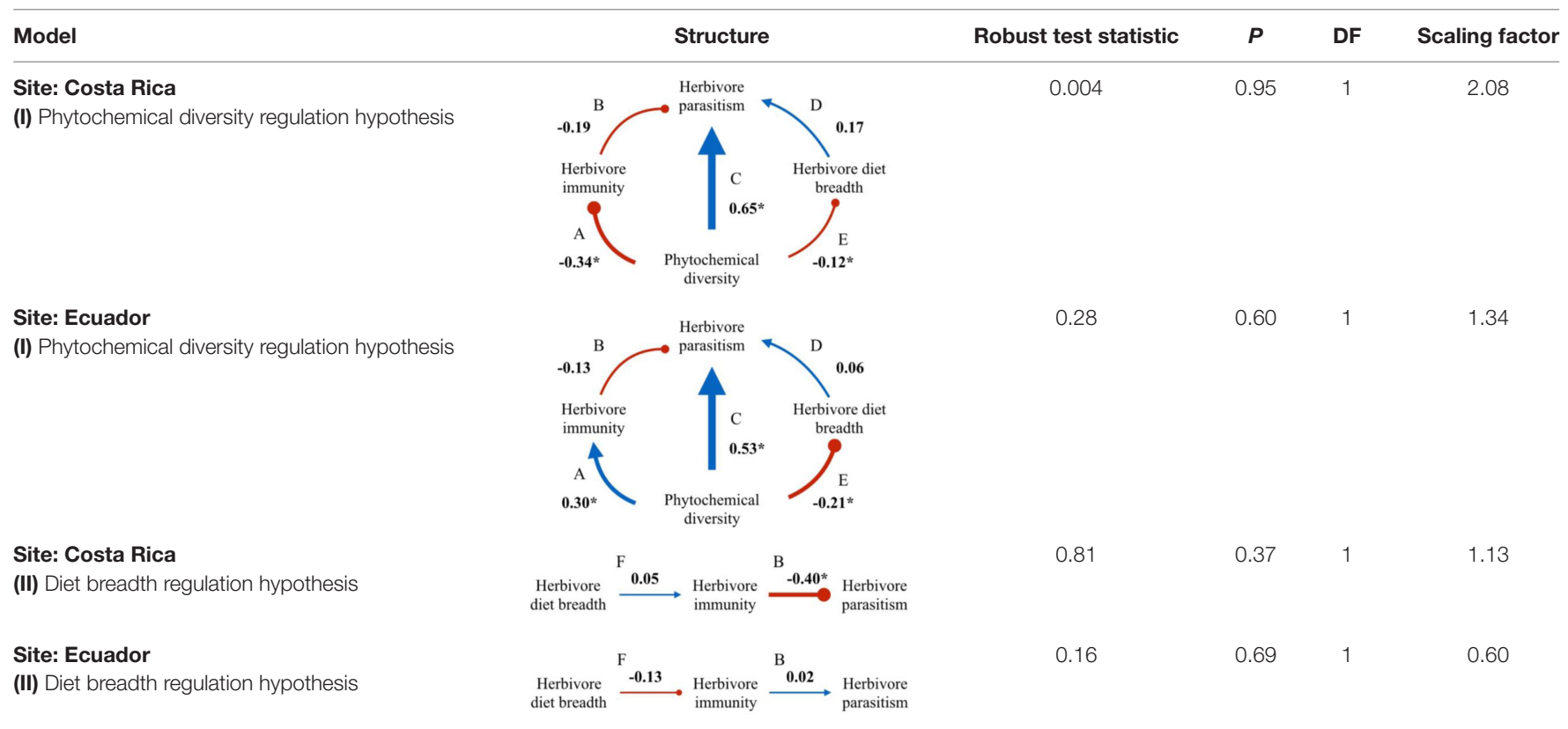

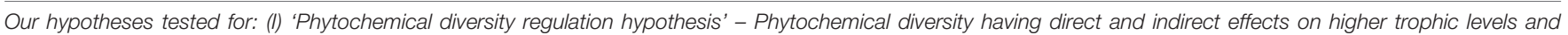

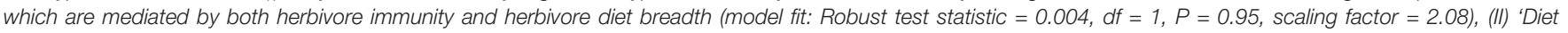

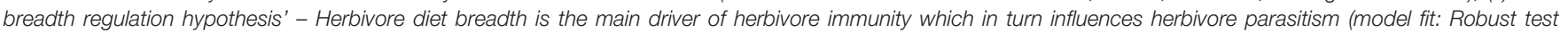

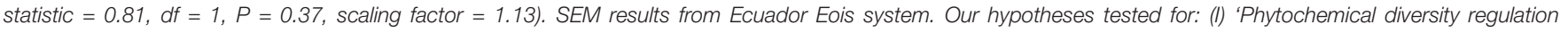

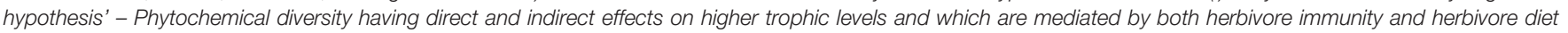

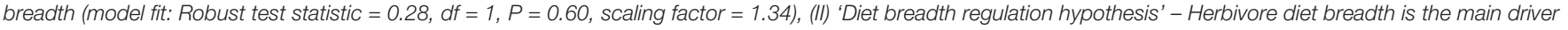

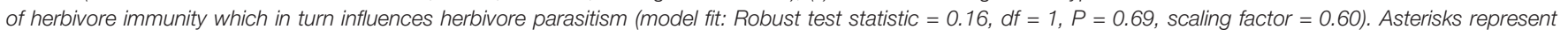
significant path coefficients $(P<0.05)$.

positive effect of phytochemical diversity on herbivore parasitism (Figure 2C, standardised path coefficient (hereafter, $\mathrm{spc}$ ) $=0.65$, $P<0.01$, slope $\left.\left(B_{1}\right)=1.13\right)$, showing that herbivores feeding on plants with high phytochemical diversity had higher parasitism rates. This model also showed that phytochemical diversity decreases herbivore immunity (Figure 2D, $\mathrm{spc}=-0.34, P<0.01$, $\left.B_{1}=-0.14\right)$. It supports the hypothesis that higher herbivore immunity decreases herbivore parasitism frequency (Figure 2B, spc $\left.=-0.19, P=0.08, B_{1}=-1.52\right)$. Lastly, this model shows a negative effect of phytochemical diversity on herbivore diet breadth (i.e., Piper species with greater phytochemical diversity are consumed by more specialised Eois species; $\mathrm{spc}=-0.12$, $\left.P=0.03, B_{1}=-2.66\right)$. In turn, herbivore diet breadth has a weak, positive effect on herbivore parasitism (i.e., generalists have higher levels of parasitism; $\left.\mathrm{spc}=0.17, P=0.11, B_{1}=0.001\right)$. The same model was strongly supported by our Ecuador Eois data, however, the directions of some of the relationships were reversed (Ecuador model fit: Robust test statistic $=0.28, \mathrm{df}=1$, $P=0.60$, scaling factor $=1.34$ ). Consistent with the Costa Rica data, phytochemical diversity has a strong positive effect on herbivore parasitism (Figure 3C, $\mathrm{spc}=0.53, P<0.01, B_{1}=1.93$ ), however, in contrast to the Costa Rica data, phytochemical diversity has a positive effect on herbivore immunity (Figure 3D, $\left.\mathrm{spc}=0.30, P<0.01, B_{1}=0.30\right)$. Phytochemical diversity has a negative effect on herbivore diet breadth $(\mathrm{spc}=-0.21$, $\left.P<0.01, B_{1}=-9.55\right)$, and herbivore immunity negatively affects herbivore parasitism (Figure 3B, spc $=-0.13, P=0.22$,
$\left.B_{1}=0.08\right)$. Lastly, diet breath has no effect on herbivore parasitism ( $\mathrm{spc}=0.06, P=0.62, B_{1}=-0.003$ ). Models for Q. cerealis caterpillars in Costa Rica did not fit the data, for example, a model where phytochemical diversity affects herbivore immunity, which in turn influences herbivore parasitism, was a poor fit to the data (model fit: Robust test statistic $=28.37$, $\mathrm{df}=1, P<0.01$, scaling factor $=0.50)$. However, a separate regression analysis showed that phytochemical diversity had a negative relationship with $Q$. cerealis parasitism $\left[B_{1}=-4.39\right.$, $\left.F_{(1,63)}=15.25, P<0.01\right]$.

\section{Model II: Diet Breadth Regulation Hypothesis}

The diet breadth regulation hypothesis (model II) is a simpler model focusing on the effects of diet breadth on herbivore immunity and parasitism (Costa Rica model fit: Robust test statistic $=0.81$, $\mathrm{df}=1, P=0.37$, scaling factor $=1.13$ ). In Costa Rica, this model shows that greater diet breadth (measured as Eois species that are documented feeding on a greater number of host plants) had a weak positive effect on herbivore immune response (Figure 2A, spc $=0.05, P=0.76$, $\left.B_{1}=0.001\right)$ and that immune function reduces parasitism success (Figure 2B, spc $=-0.40, P<0.01, B_{1}=-1.52$ ). The diet breadth regulation hypothesis was again supported by our Ecuador data (model II) (Ecuador model fit: Robust test statistic $=0.16$, df $=1, P=0.69$, scaling factor $=0.60)$, 


\section{Costa Rica}
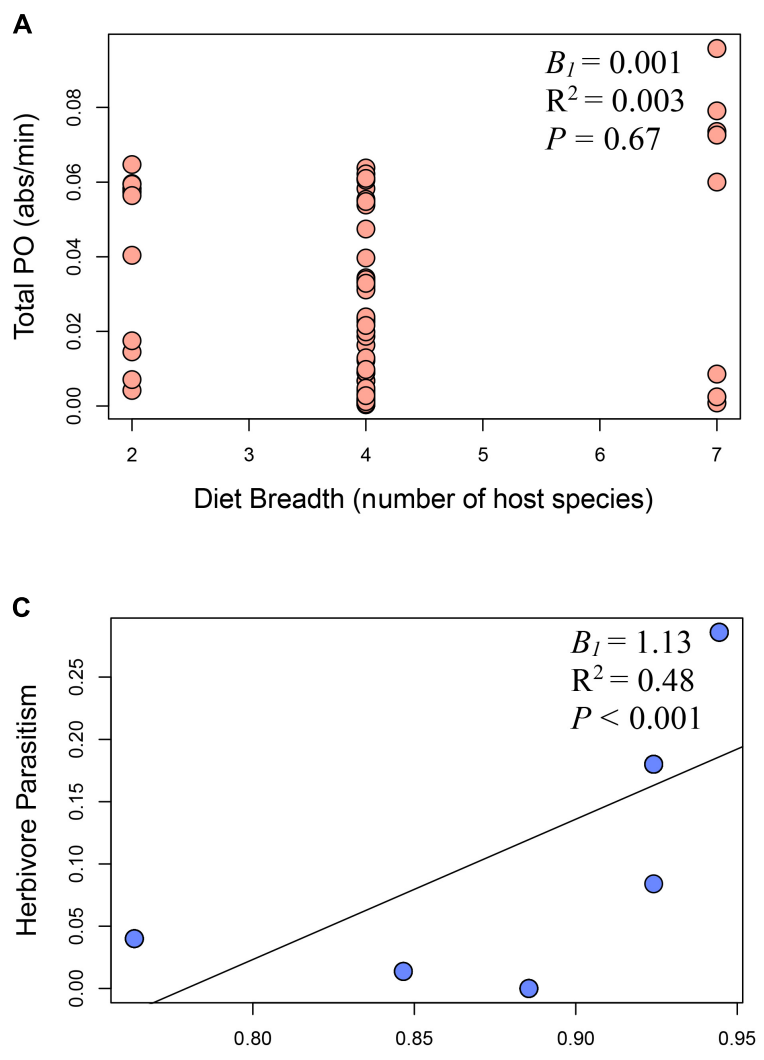

Phytochemical Diversity (NMR bin diversity)
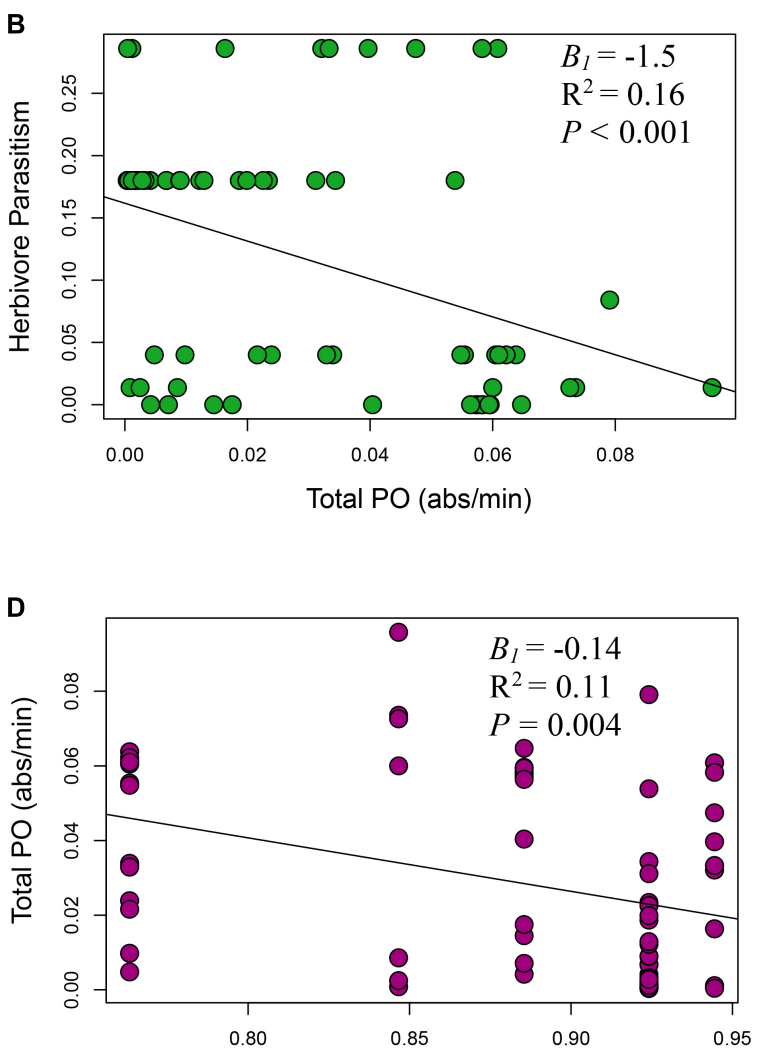

Phytochemical Diversity (NMR bin diversity)

FIGURE 2 | Multi-panel regression plots of Eois ecoimmunological parameters in Costa Rica: (A) Relationship between diet breadth, measured as number of host species, and Eois immune response, measured as total phenoloxidase absorbance per minute $\left(B_{1}=0.001, R^{2}=0.003, F_{1,68}=0.18, P=0.67\right)$. (B) Eois immune response and percent Eois parasitism $\left(B_{1}=-1.5, R^{2}=0.16, F_{1,68}=12.95, P<0.001\right)$. (C) Phytochemical diversity, measured as NMR binned peak diversity, and Eois percent parasitism $\left(B_{1}=1.13, R^{2}=0.48, F_{1,68}=63.78, P<0.001\right)$. (D) Phytochemical diversity and Eois immune response $\left(B_{1}=-0.14, R^{2}=0.11\right.$, $\left.F_{1,68}=8.67, P=0.004\right)$.

but for this site, a greater diet breadth had a weak negative association with herbivore immunity (Figure $\mathbf{3 A}, \mathrm{spc}=-0.13$, $\left.P=0.30, B_{1}=-0.003\right)$, and herbivore immunity has no effect on herbivore parasitism (Figure 3B, $\mathrm{spc}=0.02, P=0.84$, $\left.B_{1}=0.08\right)$.

\section{DISCUSSION}

Our results corroborate many other studies demonstrating that the chemistry of herbivore host plants, as well as herbivore diet breadth have strong effects on multiple aspects of herbivore ecology (Berenbaum and Neal, 1985; Haviola et al., 2007; Diamond and Kingsolver, 2011; Lampert and Bowers, 2015), including immunity and parasitism (Smilanich et al., 2009a; Hansen et al., 2017). A focus on the immune response allows for investigation of an important physiological parameter that is directly linked to protection against natural enemies (Smilanich et al., 2009b), putting our results in a strong tri-tropic context. It is also interesting that the relationships between phytochemical diversity, immunity, and parasitism were dependent upon the diet breadth of the specialist herbivores and that relationships varied across herbivore taxa and site. In Costa Rica, Eois feeding on Piper species with high phytochemical diversity had a weakened immune response, while the immune response of $Q$. cerealis was unaffected. It is important to note that the sample size for some herbivore species in Ecuador was small, which weakens the strength of our results (Table 2). For instance, three different herbivore species were only collected once (Table 2) - collecting many replicate herbivore species in the tropics can be difficult, depending on their abundance and distribution. Nevertheless, our results correspond with the importance of diet breadth in other results with this system (Richards et al., 2015). Eois data in Ecuador fit the same two models as in Costa Rica, however, some relationships were reversed. For example, in Costa Rica, individuals with a strong immune response had lower parasitism frequency (model II), however, in Ecuador herbivore immunity had almost no effect on parasitism frequency. This difference may be due to the differences in parasitoid pressure between the two sites. 


\section{Ecuador}

A
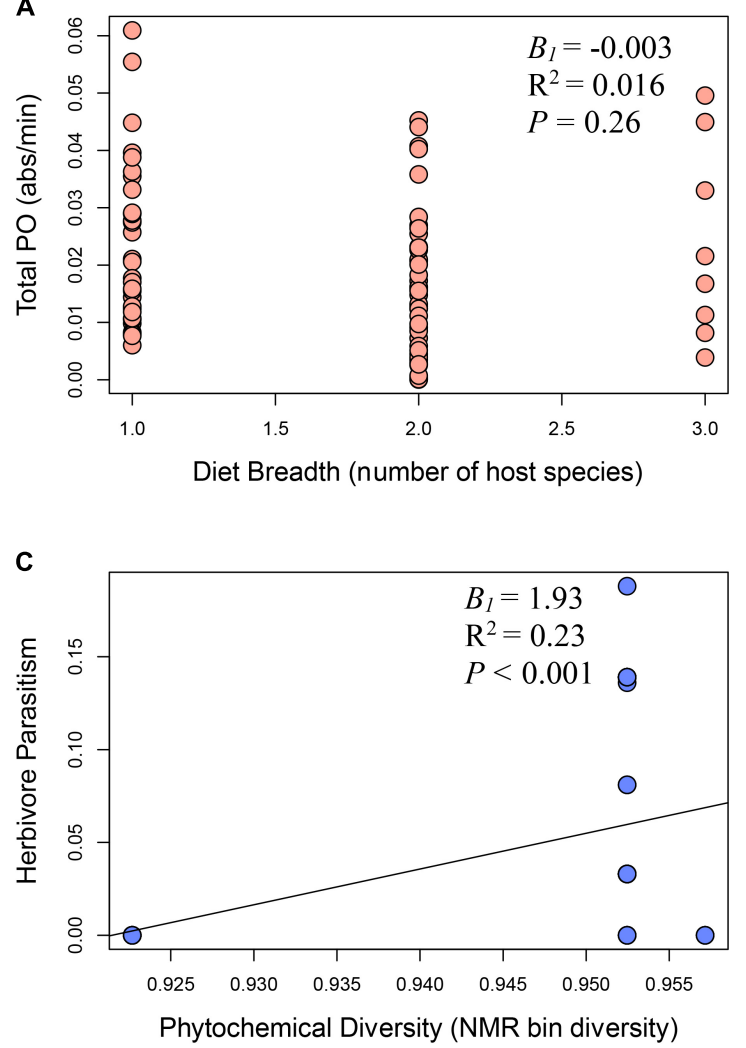

B

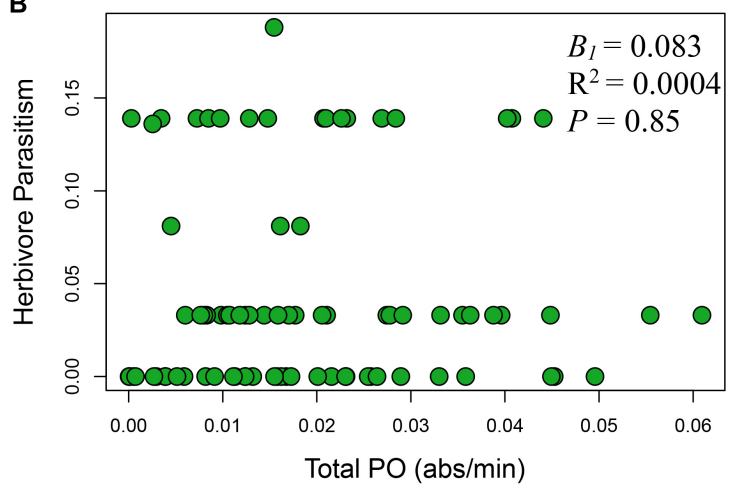

D

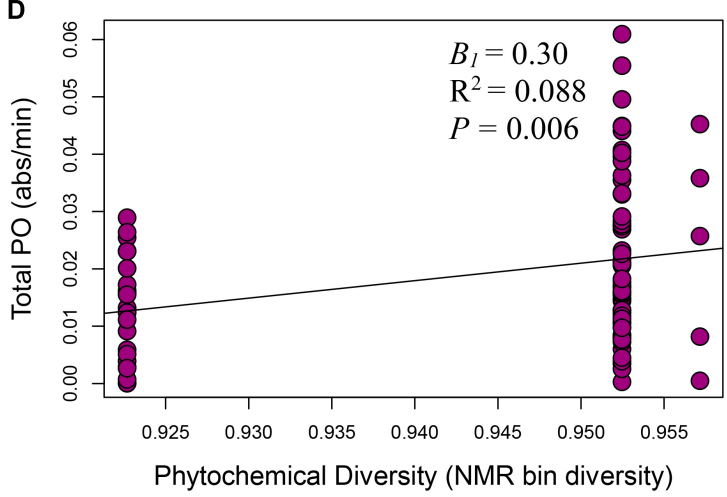

FIGURE 3 | Multi-panel regression plots of Eois ecoimmunological parameters in Ecuador: (A) Relationship between diet breadth, measured as number of host species, and Eois immune response, measured as total phenoloxidase absorbance per minute $\left(B_{1}=-0.003, R^{2}=0.016, F_{1,81}=1.28, P=0.26\right)$. (B) Eois immune response and percent Eois parasitism $\left(B_{1}=0.083, R^{2}=0.0004, F_{1,81}=0.036, P=0.85\right)$. (C) Phytochemical diversity, measured as NMR binned peak diversity, and Eois percent parasitism $\left(B_{1}=1.93, R^{2}=0.23, F_{1,81}=23.82, P<0.001\right)$. (D) Phytochemical diversity and Eois immune response $\left(B_{1}=0.30, R^{2}=0.088\right.$, $\left.F_{1,81}=7.82, P=0.006\right)$.

Compared to Ecuador, the database shows that Eois in Costa Rica have three times more parasitism by a relatively more specialised parasitoid community (Braconidae). Our Ecuador data include plant-caterpillar species pairs that are not well represented in our historical database and which have $0 \%$ parasitism as a result. We ruled out that this was driving our observed patterns by re-running our SEMs without plantcaterpillar species pairs that had low representation in our database, but found the same qualitative result. We therefore included these data points in our final analysis. Other possible particulars of the taxa and sites used for our study, such as degree of specialisation and elevation of the site, may also be responsible for these differences, but greater insight into those variables will require further experimentation using carefully selected taxa and locations.

Untangling relationships between plant chemistry, herbivores, and natural enemies has been a focus of insect ecology for decades (Price et al., 1980; Bernays and Graham, 1988; Dyer, 1995, 2011) and our results with Eois in Costa Rica are consistent with emerging paradigms of the importance of phytochemistry in mediating multi-trophic interactions. Most notably, we provide further support for the 'safe haven hypothesis' (Lampert et al., 2010) and the 'vulnerable host hypothesis' (Smilanich et al., 2009a). Eois data from Costa Rica support all aspects of this 'safe haven hypothesis' and data from both sites support the more general concept that changes in chemistry are likely to alter herbivore immunity and parasitism the positive effects of phytochemical diversity on herbivore immunity in Ecuador are not inconsistent with this hypothesis, and they simply require further investigation to determine mechanisms causing this relationship. Furthermore, both SEM models (Table 4, Hypotheses I and II) are consistent with the growing body of evidence that the ability of an insect herbivore to mount an immune response is negatively associated with herbivore parasitism (Bukovinszky et al., 2009; Quintero et al., 2014), which is an important component of the 'safe haven hypothesis,' and some have argued that this is the best predictor of parasitism (Smilanich et al., 2009b; Greeney et al., 2012).

Other studies that support the 'safe haven hypothesis' (Gentry and Dyer, 2002; Lampert et al., 2010) or related hypotheses (i.e., 'nasty host hypothesis' Barbosa et al., 1991; Gauld et al., 1992) 
have focused on detoxification or sequestration of individual compounds or entire classes of compounds and have measured relative concentrations of those compounds (e.g., Haviola et al., 2007; Smilanich et al., 2009a; Lampert and Bowers, 2015). We utilise a different approach and consider the fact that phytochemical mixtures are complex and herbivores may be as susceptible to mixture complexity, synergies, or additive effects rather than just increases in concentrations of individual compounds or classes, such as tannins (Richards et al., 2015). One shortcoming of this approach is that results will require further investigation to get at mechanism. In Costa Rica, the immune responses of Eois species were negatively affected by increases in phytochemical diversity (Table 4, Hypothesis I). Another study with Eois on Piper found that changes in mixture complexity are associated with synergistic effects on parasitoid success (Richards et al., 2010). It is possible that host plants with higher phytochemical diversity are more likely to have synergistic effects on herbivores, impairing immune function, regardless of whether the mixtures are sequestered.

It is interesting to note that the results depended on taxon (Quadrus versus Eois) and site (Ecuador versus Costa Rica). Such variation is expected, and it is worth further investigation to determine conditions that are favourable for these chemically mediated tri-tropic interactions. Site and taxon were treated as random effects in the broader sense and were not statistically compared; nevertheless, it is interesting to consider possibilities for some of the differences across the two taxa and the two sites. Specialist Eois caterpillars in Costa Rica support our predictions, whereas, the same genus of caterpillars in Ecuador do not support any of our a priori models. Elevation is one clear difference between these sites, with the cloud forest in Ecuador situated 2,000 $\mathrm{m}$ higher than the lowland forest in Costa Rica. It is well known that herbivore development rates, herbivory, levels of predation, and herbivore diversity are lower at higher elevations, while parasitism and parasitoid diversity increase with elevation (Rodríguez-Castañeda et al., 2011, 2016), so it is not surprising that the specifics of chemically mediated tri-tropic interactions would vary with elevation. Reasons for the positive effect of phytochemical diversity on immunity at higher elevation are not obvious, but given the higher levels of parasitism and slow development rates, it is possible that maximised immunity is enhanced with slow development rates since larvae are exposed to parasitoids for longer periods of time. Similarly, there are many differences between the geometrid and hesperiid caterpillars utilised in our study, including diet breadth; however, one large difference is that Quadrus is a concealed feeder, and concealed feeders are affected less by phytochemical defence (Sandberg and Berenbaum, 1989; Berenbaum, 1990) and experience very high levels of parasitism (Gentry and Dyer, 2002). As such, Q. cerealis appeared to be unaffected by changes in chemistry and experienced extremely high levels of parasitism. There are likely unmeasured variables that influence immunity of hesperiids and more generally of concealed feeders, and it is certainly possible that the greater diet breadth played a role in the differences noted here.

In summary, our research builds on previous work investigating the effects of phytochemical diversity and herbivore diet breadth on ecoimmunology and tri-trophic interactions. These results support the hypothesis that variation in phytochemical diversity, rather than individual compounds, was a predictor of tri-trophic interactions and herbivore immunity (Richards et al., 2010, 2016). These patterns are also particularly important for understanding tropical systems, which are typically characterised by intense biotic interactions and high levels of diversity (Dyer and Coley, 2002; Novotny et al., 2006). Future work should investigate how much intraspecific phytochemical variation exists within these Piper species, how intraspecific variation compares across different Piper species, and what is driving that variation. Further, how does this intraspecific variation affect higher trophic levels and what are the differences in responses between predators and parasitoids. A field experiment evaluating the susceptibility of herbivores to parasitoids feeding on Piper of varying phytochemical profiles would greatly add to our understanding of the consequences of phytochemical diversity on herbivore immunity. Lastly, as the effects of global change worsen, including loss of tropical forests, the diversity of plant secondary metabolites is predicted to decrease, and understanding this diversity is a key part of documenting these losses.

\section{AUTHOR CONTRIBUTIONS}

LR, AS, and LD designed the experiments. HS performed the field work. HS and LD analysed the data. LD, LR, AS, and PH provided advice for the data analysis. HS wrote the first drafts. All authors contributed to additional draft of the manuscript.

\section{FUNDING}

This work was funded by NSF (DEB-1442103; IOS-1456354) and by a generous donation to the Hitchcock Fund for Chemical Ecology Research. HS was funded by NSERC during the manuscript preparation.

\section{ACKNOWLEDGMENTS}

We thank to Matt Forister and Zach Marion for statistical advice. Many thanks also go to our technicians Humberto Garcia (Costa Rica) and Wilmer Simbaña (Ecuador) for their year-round work without which this manuscript would not be possible. We would also like to thank Natalia Rico, Ron Parry, Andrea Glassmire, and Josh Snook for help with data collection. Finally, we acknowledge and thank the Earthwatch Institute and the many volunteers that have helped build our databases in Costa Rica and Ecuador.

\section{SUPPLEMENTARY MATERIAL}

The Supplementary Material for this article can be found online at: https://www.frontiersin.org/articles/10.3389/fpls.2018.00656/ full\#supplementary-material 


\section{REFERENCES}

Adamo, S. A. (2004). Estimating disease resistance in insects: phenoloxidase and lysozyme-like activity and disease resistance in the cricket Gryllus texensis. J. Insect Physiol. 50, 209-216. doi: 10.1016/j.jinsphys.2003.11.011

Agrawal, A. A. (2000). Mechanisms, ecological consequences and agricultural implications of tri-trophic interactions. Curr. Opin. Plant Biol. 3, 329-335. doi: 10.1016/S1369-5266(00)00089-3

Barbosa, P., Gross, P., and Kemper, J. (1991). Influence of plant allelochemicals on the tobacco hornworm and its parasitoid, Cotesia Congregata. Ecology 72, 1567-1575. doi: 10.2307/1940956

Barbour, M. A., Rodriguez-Cabal, M. A., Wu, E. T., Julkunen-Tiitto, R., Ritland, C. E., Miscampbell, A. E., et al. (2015). Multiple plant traits shape the genetic basis of herbivore community assembly. Funct. Ecol. 29, 995-1006. doi: 10.1111/ 1365-2435.12409

Becerra, J. X. (2007). The impact of herbivore-plant coevolution on plant community structure. Proc. Natl. Acad. Sci. U.S.A. 104, 7483-7488. doi: 10. 1073/pnas.0608253104

Becerra, J. X. (2015). On the factors that promote the diversity of herbivorous insects and plants in tropical forests. Proc. Natl. Acad. Sci. U.S.A. 112, 6098-6103. doi: 10.1073/pnas. 1418643112

Beckage, N. E. (2008). Insect Immunology. Oxford: Academic Press.

Berenbaum, M. (1990). Evolution of specialization in insect-umbellifer associations. Annu. Rev. Entomol. 35, 319-343. doi: 10.1146/annurev.en. 35.010190 .001535

Berenbaum, M., and Neal, J. J. (1985). Synergism between myristicin and xanthotoxin, a naturally cooccurring plant toxicant. J. Chem. Ecol. 11, 1349-1358. doi: 10.1007/BF01012136

Bernays, E. A., and Graham, M. (1988). On the evolution of host specificity in phytophagous arthropods. Ecology 69, 886-892. doi: 10.2307/1941237

Brehm, G., Bodner, F., Strutzenberger, P., Hünefeld, F., and Fiedler, K. (2011). Neotropical Eois (Lepidoptera: Geometridae): checklist, biogeography, diversity, and description patterns. Ann. Entomol. Soc. Am. 104, 1091-1107. doi: 10.1603/AN10050

Bukovinszky, T., Poelman, E. H., Gols, R., Prekatsakis, G., Vet, L. E. M., Harvey, J. A., et al. (2009). Consequences of constitutive and induced variation in plant nutritional quality for immune defence of a herbivore against parasitism. Oecologia 160, 299-308. doi: 10.1007/s00442-009-1308-y

Carvalheiro, L. G., Buckley, Y. M., and Memmott, J. (2010). Diet breadth influences how the impact of invasive plants is propagated through food webs. Ecology 91, 1063-1074. doi: 10.1890/08-2092.1

Cerenius, L., Lee, B. L., and Söderhäll, K. (2008). The proPO-system: pros and cons for its role in invertebrate immunity. Trends Immunol. 29, 263-271. doi: 10.1016/j.it.2008.02.009

Coley, P. D., and Barone, J. A. (1996). Herbivory and plant defenses in tropical forests. Annu. Rev. Ecol. Syst. 27, 305-335. doi: 10.1146/annurev.ecolsys. 27.1.305

Coley, P. D., Bateman, M. L., and Kursar, T. A. (2006). The effects of plant quality on caterpillar growth and defense against natural enemies. Oikos 115, 219-228. doi: 10.1111/j.2006.0030-1299.14928.x

Connahs, H., Rodríguez-Castañeda, G., Walters, T., Walla, T., and Dyer, L. (2009). Geographic variation in host-specificity and parasitoid pressure of an herbivore (Geometridae) associated with the tropical genus Piper (Piperaceae). J. Insect. Sci. 9:28. doi: 10.1673/031.009.2801

Crawford, K. M., Crutsinger, G. M., and Sanders, N. J. (2007). Host-plant genotypic diversity mediates the distribution of an ecosystem engineer. Ecology 88, 2114-2120. doi: 10.1890/06-1441.1

Crutsinger, G. M., Collins, M. D., Fordyce, J. A., Gompert, Z., Nice, C. C., and Sanders, N. J. (2006). Plant genotypic diversity predicts community structure and governs an ecosystem process. Science 313, 966-968. doi: 10.1126/science. 1128326

Cunningham, S. A., Summerhayes, B., and Westoby, M. (1999). Evolutionary divergences in leaf structure and chemistry, comparing rainfall and soil nutrient gradients. Ecology 69, 569-588. doi: 10.1890/0012-9615(1999) 069[0569:EDILSA]2.0.CO;2

Diamond, S. E., and Kingsolver, J. G. (2011). Host plant quality, selection history and trade-offs shape the immune responses of Manduca sexta. Proc. R. Soc. Lond. B Biol. Sci. 278, 289-297. doi: 10.1098/rspb.2010.1137
Dyer, L. A. (1995). Tasty generalists and nasty specialists? Antipredator mechanisms in tropical Lepidopteran larvae. Ecology 76, 1483-1496. doi: 10. 2307/1938150

Dyer, L. A. (2011). New synthesis-back to the future: new approaches and directions in chemical studies of coevolution. J. Chem. Ecol. 37:669. doi: 10. 1007/s10886-011-9979-3

Dyer, L. A., and Coley, P. D. (2002). "Tri-trophic interactions in tropical versus temperate communities," in Multitrophic Level Interactions, eds T. Tscharntke and B. Hawkins (Cambridge: Cambridge University Press), 67-88.

Dyer, L. A., Dodson, C. D., Stireman, J. O., Smilanich, A. M., Fincher, R. M., and Letourneau, D. K. (2003). Synergistic effects of three Piper amides on generalist and specialist herbivores. J. Chem. Ecol. 29, 2499-2514. doi: 10.1023/ A:1026310001958

Dyer, L. A., and Floyd, T. (1993). Determinants of predation on phytophagous insects: the importance of diet breadth. Oecologia 96, 575-582. doi: 10.1007/ BF00320516

Dyer, L. A., and Gentry, G. (1999). Predicting natural-enemy responses to herbivores in natural and managed systems. Ecol. Appl. 9, 402-408. doi: 10.1890/1051-0761(1999)009[0402:PNERTH]2.0.CO;2

Dyer, L. A., Gentry, G. L., Greeney, H. F., and Walla, T. W. (2010). Caterpillars and Parasitoids of the Eastern Andes in Ecuador. Available at: http://www. caterpillars.org [Accessed January 16, 2018].

Dyer, L. A., Letourneau, D. K., Dodson, C. D., Tobler, M. A., Stireman, J. O., and Hsu, A. (2004). Ecological causes and consequences of variation in defensive chemistry of a neotropical shrub. Ecology 85, 2795-2803. doi: 10.1890/03-0233

Dyer, L. A., and Palmer, A. N. (2004). Piper: A Model Genus for Studies of Phytochemistry, Ecology and Evolution. New York, NY: Kluwer Academic Publishers. doi: 10.1007/978-0-387-30599-8

Dyer, L. A., Singer, M. S., Lill, J. T., Stireman, J. O., Gentry, G. L., Marquis, R. J., et al. (2007). Host specificity of Lepidoptera in tropical and temperate forests. Nature 448, 696-699. doi: 10.1038/nature05884

Ehrlich, P. R., and Raven, P. H. (1964). Butterflies and plants: a study in coevolution. Evolution 18, 586-608. doi: 10.1111/j.1558-5646.1964.tb0 1674.x

Fraenkel, G. S. (1959). The raison d'Etre of secondary plant substances. Science 129, 1466-1470. doi: 10.1126/science.129.3361.1466

Gauld, I. D., Gaston, K. J., and Janzen, D. H. (1992). Plant allelochemicals, tritrophic interactions and the anomalous diversity of tropical parasitoids: the "nasty" host hypothesis. Oikos 65, 353-357. doi: 10.2307/3545032

Gentry, G. L., and Dyer, L. A. (2002). On the conditional nature of neotropical caterpillar defenses against their natural enemies. Ecology 83, 3108-3119. doi: 10.1890/0012-9658(2002)083[3108:OTCNON]2.0.CO;2

Glassmire, A. E., Jeffrey, C. S., Forister, M. L., Parchman, T. L., Nice, C. C., Jahner, J. P., et al. (2016). Intraspecific phytochemical variation shapes community and population structure for specialist caterpillars. New Phytol. 212, 208-219. doi: $10.1111 /$ nph. 14038

González-Santoyo, I., and Córdoba-Aguilar, A. (2012). Phenoloxidase: a key component of the insect immune system. Entomol. Exp. Appl. 142, 1-16. doi: 10.1111/j.1570-7458.2011.01187.x

Greeney, H. F., Dyer, L., and Smilanich, M. (2012). Feeding by lepidopteran larvae is dangerous: a review of caterpillars' chemical, physiological, morphological, and behavioral defenses against natural enemies. Invertebrate Surviv. J. 9, 7-34.

Greig, N. (1993). Predispersal seed predation on 5 Piper species in tropical rainforest. Oecologia 93, 412-420. doi: 10.1007/BF00317886

Hairston, N. G., Smith, F. E., and Slobodkin, L. B. (1960). Community structure, population control and competition. Am. Nat. 94, 421-425. doi: 10.1086/282146

Hansen, A. C., Glassmire, A. E., Dyer, L. A., and Smilanich, A. M. (2017). Patterns in parasitism frequency explained by diet and immunity. Ecography 40, 803-805. doi: 10.1111/ecog.02498

Haviola, S., Kapari, L., Ossipov, V., Rantala, M. J., Ruuhola, T., and Haukioja, E. (2007). Foliar phenolics are differently associated with Epirrita autumnata growth and immunocompetence. J. Chem. Ecol. 33, 1013-1023. doi: 10.1007/ s10886-007-9271-8

Hay, M. E., Kappel, Q. E., and Fenical, W. (1994). Synergisms in plant defenses against herbivores - interactions of chemistry, calcification, and plant-quality. Ecology 75, 1714-1726. doi: 10.2307/1939631

Hunter, M. D. (2016). The Phytochemical Landscape: Linking Trophic Interactions and Nutrient Dynamics. Princeton, NJ: Princeton University Press. 
Hunter, M. D., and Price, P. W. (1992). Playing chutes and ladders: heterogeneity and the relative roles of bottom-up and top-down forces in natural communities. Ecology 73, 724-732.

Ives, A. R., Cardinale, B. J., and Snyder, W. E. (2005). A synthesis of subdisciplines: predator-prey interactions, and biodiversity and ecosystem functioning. Ecol. Lett. 8, 102-116. doi: 10.1111/j.1461-0248.2004.00698.x

Johnson, M. T. J. (2008). Bottom-up effects of plant genotype on aphids, ants, and predators. Ecology 89, 145-154. doi: 10.1890/07-0395.1

Jones, C. G., and Firn, R. (1991). On the evolution of plant secondary chemical diversity. Philos. Trans. R. Soc. B Biol. Sci. 333, 273-280. doi: 10.1098/rstb.1991. 0077

Lampert, E. (2012). Influences of plant traits on immune responses of specialist and generalist herbivores. Insects 3, 573-592. doi: 10.3390/insects3020573

Lampert, E. C., and Bowers, M. D. (2015). Incompatibility between plant-derived defensive chemistry and immune response of two Sphingid herbivores. J. Chem. Ecol. 41, 85-92. doi: 10.1007/s10886-014-0532-Z

Lampert, E. C., Dyer, L. A., and Bowers, M. D. (2010). Caterpillar chemical defense and parasitoid success: Cotesia congregata parasitism of Ceratomia catalpae. J. Chem. Ecol. 36, 992-998. doi: 10.1007/s10886-010-9840-0

Lampert, E. C., Zangerl, A. R., Berenbaum, M. R., and Ode, P. J. (2011). Generalist and specialist host-parasitoid associations respond differently to wild parsnip (Pastinaca sativa) defensive chemistry. Ecol. Entomol. 36, 52-61. doi: 10.1111/j. 1365-2311.2010.01244.x

Lefcheck, J. S. (2016). piecewiseSEM: piecewise structural equation modelling in $\mathrm{r}$ for ecology, evolution, and systematics. Methods Ecol. Evol. 7, 573-579. doi: 10.1111/2041-210X.12512

Lill, J. T., Marquis, R. J., and Ricklefs, R. E. (2002). Host plants influence parasitism of forest caterpillar. Nature 417, 170-173. doi: 10.1038/417170a

Marquis, R. J. (1991). "Herbivore fauna of Piper (Piperaceae) in a Costa Rican wet forest: diversity, specificity, and impact," in Plant-Animal Interactions: Evolutionary Ecology in Tropical and Temperate Regions, eds P. W. Price, T. M. Lewinsohn, G. W. Fernandes, and W. W. Benson (New York, NY: John Wiley \& Sons), 179-208.

Novotny, V., Drozd, P., Miller, S. E., Kulfan, M., Janda, M., Basset, Y., et al. (2006). Why are there so many species of herbivorous insects in tropical rainforests? Science 313, 1115-1118.

O'Connor, M. I., Gonzalez, A., Byrnes, J. E. K., Cardinale, B. J., Duffy, J. E., Gamfeldt, L., et al. (2016). A general biodiversity-function relationship is mediated by trophic level. Oikos 126, 18-31. doi: 10.1111/oik.03652

Price, P. W. (2002). Resource-driven terrestrial interaction webs. Ecol. Res. 17, 241-247. doi: 10.1038/nature11131

Price, P. W., Bouton, C. E., Gross, P., McPheron, B. A., Thompson, J. N., and Weis, A. E. (1980). Interactions among three trophic levels: influence of plants on interactions between insect herbivores and natural enemies. Annu. Rev. Ecol. Syst. 11, 41-65. doi: 10.1146/annurev.es.11.110180.000353

Quintero, C., Lampert, E. C., and Bowers, M. D. (2014). Time is of the essence: direct and indirect effects of plant ontogenetic trajectories on higher trophic levels. Ecology 95, 2589-2602. doi: 10.1890/13-2249.1

R Core Team (2017). R: A Language and Environment for Statistical Computing. Vienna: R Foundation for Statistical Computing. Available at: https://www.rproject.org/

Reudler, J. H., Biere, A., Harvey, J. A., and van Nouhuys, S. (2011). Differential performance of a specialist and two generalist herbivores and their parasitoids on Plantago lanceolata. J. Chem. Ecol. 37, 765-778. doi: 10.1007/s10886-0119983-7

Richards, L. A., Dyer, L. A., Forister, M. L., Smilanich, A. M., Dodson, C. D., Leonard, M. D., et al. (2015). Phytochemical diversity drives plant-insect community diversity. Proc. Natl. Acad. Sci. U.S.A. 112, 10973-10978. doi: 10. 1073/pnas. 1504977112

Richards, L. A., Dyer, L. A., Smilanich, A. M., and Dodson, C. D. (2010). Synergistic effects of amides from two Piper species on generalist and specialist herbivores. J. Chem. Ecol. 36, 1105-1113. doi: 10.1007/s10886-010-9852-9

Richards, L. A., Glassmire, A. E., Ochsenrider, K. M., Smilanich, A. M., Dodson, C. D., Jeffrey, C. S., et al. (2016). Phytochemical diversity and synergistic effects on herbivores. Phytochem. Rev. 15, 1153-1166. doi: 10.1007/s11101-016-9479-8

Richards, L. A., Lampert, E. C., Bowers, M. D., Dodson, C. D., Smilanich, A. M., and Dyer, L. A. (2012). Synergistic effects of iridoid glycosides on the survival, development and immune response of a specialist caterpillar, Junonia coenia (Nymphalidae). J. Chem. Ecol. 38, 1276-1284. doi: 10.1007/s10886-012-0190-y Rodríguez-Castañeda, G., Brehm, G., Fiedler, K., and Dyer, L. (2016). Ant predation on herbivores through a multitrophic lens: how effects of ants on plant herbivore defense and natural enemies vary along temperature gradients. Curr. Opin. Insect Sci. 14, 73-80. doi: 10.1016/j.cois.2016.02.001

Rodríguez-Castañeda, G., Dyer, L. A., Brehm, G., Connahs, H., Forkner, R. E., and Walla, T. R. (2010). Tropical forests are not flat: how mountains affect herbivore diversity. Ecol. Lett. 13, 1348-1357. doi: 10.1111/j.1461-0248.2010.01525.x

Rodríguez-Castañeda, G., Forkner, R. E., Tepe, E. J., Gentry, G. L., and Dyer, L. A. (2011). Weighing defensive and nutritive roles of ant mutualists across a tropical altitudinal gradient. Biotropica 43, 343-350. doi: 10.1111/j.1744-7429. 2010.00700.x

Rosseel, Y., Oberski, D., Byrnes, J., Vanbrabant, L., Savalei, V., Merkle, E., et al. (2017). Package 'lavaan'. Available at: https://cran.r-project.org/package=lavaan [accessed January 16, 2018].

Salazar, D., Jaramillo, A., and Marquis, R. J. (2016). The impact of plant chemical diversity on plant - herbivore interactions at the community level. Oecologia 181, 1199-1208. doi: 10.1007/s00442-016-3629-y

Sandberg, S. L., and Berenbaum, M. R. (1989). Leaf-tying by tortricid larvae as an adaptation for feeding on phototoxic Hypericum perforatum. J. Chem. Ecol. 15, 875-885. doi: 10.1007/BF01015183

Schoonhoven, L. M., Van Loon, J. J., and Dicke, M. (2005). Insect-Plant Biology, 2nd Edn. Oxford: Oxford University Press.

Singer, M. S., Mason, P. A., and Smilanich, A. M. (2014). Ecological immunology mediated by diet in herbivorous insects. Integr. Comp. Biol. 54, 913-921. doi: 10.1093/icb/icu089

Singer, M. S., and Stireman, J. O. (2005). The tri-trophic niche concept and adaptive radiation of phytophagous insects. Ecol. Lett. 8, 1247-1255. doi: 10.1111/j.14610248.2005.00835.x

Smilanich, A. M., and Dyer, L. A. (2012). Effects of banana plantation pesticides on the immune response of lepidopteran larvae and their parasitoid natural enemies. Insects 3, 616-628. doi: 10.3390/insects3030616

Smilanich, A. M., Dyer, L. A., Chambers, J. Q., and Bowers, M. D. (2009a). Immunological cost of chemical defence and the evolution of herbivore diet breadth. Ecol. Lett. 12, 612-621. doi: 10.1111/j.1461-0248.2009. 01309.x

Smilanich, A. M., Dyer, L. A., and Gentry, G. L. (2009b). The insect immune response and other putative defenses as effective predictors of parasitism. Ecology 90, 1434-1440.

Smilanich, A. M., Fincher, R. M., and Dyer, L. A. (2016). Does plant apparency matter? Thirty years of data provide limited support but reveal clear patterns of the effects of plant chemistry on herbivores. New Phytol. 210, 1044-1057. doi: $10.1111 /$ nph.13875

Smilanich, A. M., Vargas, J., Dyer, L. A., and Bowers, M. D. (2011). Effects of ingested secondary metabolites on the immune response of a polyphagous caterpillar Grammia incorrupta. J. Chem. Ecol. 37, 239-245. doi: 10.1007/ s10886-011-9924-5

Sternberg, E. D., Lef, T., Li, J., Lopez, C., de Castillejo, F., Li, H., et al. (2012). Food plant-derived disease tolerance and resistance in a natural butterfly-plantparasite interactions. Evolution 66, 3367-3377. doi: 10.1111/j.1558-5646.2012. 01693.x

Whitham, T. G., Bailey, J. K., Schweitzer, J. A., Shuster, S. M., Bangert, R. K., LeRoy, C. J., et al. (2006). A framework for community and ecosystem genetics: from genes to ecosystems. Nat. Rev. Genet. 7, 510-523. doi: 10.1038/nrg1877

Conflict of Interest Statement: The authors declare that the research was conducted in the absence of any commercial or financial relationships that could be construed as a potential conflict of interest.

Copyright (c) 2018 Slinn, Richards, Dyer, Hurtado and Smilanich. This is an openaccess article distributed under the terms of the Creative Commons Attribution License (CC BY). The use, distribution or reproduction in other forums is permitted, provided the original author(s) and the copyright owner are credited and that the original publication in this journal is cited, in accordance with accepted academic practice. No use, distribution or reproduction is permitted which does not comply with these terms. 